
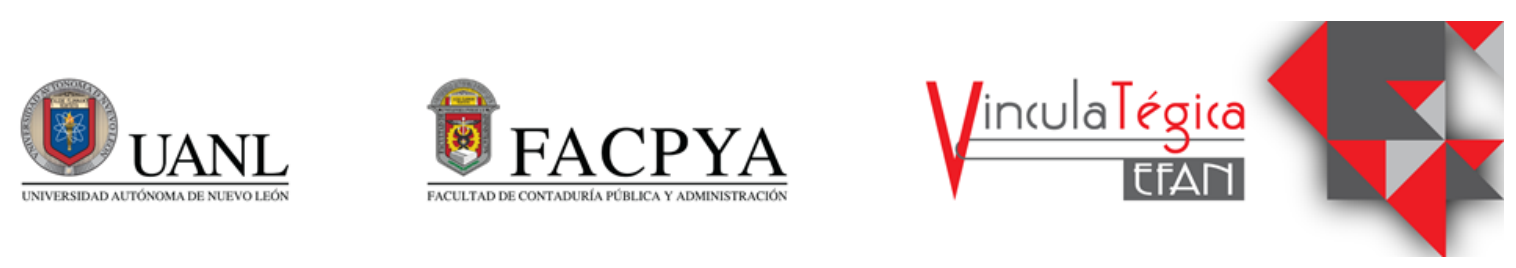

\title{
Evolución del gasto público del sector educativo de México
}

\author{
Abigail Contreras López ${ }^{1}$, Lauren Patricia López Garrido² y Artermio Jiménez Rico ${ }^{3}$ \\ 1Universidad de Guanajuato, División de Ciencias Económico Administrativas, Guanajuato, Guanajuato, \\ México, a.contreras.lopez@ugto.mx Establo S/N Col. Fraccionamiento 1, (+52) 4731451604 \\ ${ }^{2}$ Universidad de Guanajuato, División de Ciencias Económico Administrativas, Guanajuato, Guanajuato, \\ México, lp.lopezgarrido@ugto.mx Establo S/N Col. Fraccionamiento 1, (+52) 4761186447 \\ ${ }^{3}$ Universidad de Guanajuato, División de Ciencias Económico Administrativas, Guanajuato, Guanajuato, \\ México, a.jimenezrico@ugto.mx, Establo S/N Col. Fraccionamiento 1, (+52) 4731076097
}

Información del artículo revisado por pares

Fecha de aceptación: junio-2021

Fecha de publicación en línea: diciembre-2021

DOI: https://doi.org/10.29105/vtga7.1-100

\section{Resumen}

Los recursos destinados al sector educativo son una inversión para México, porque gracias a ello se impulsa el desarrollo del país. En el presente artículo se mide la evolución del Gasto Nacional Educativo (GNE) en el sector educativo en México tomando como punto de comparación el porcentaje del Producto Interno Bruto que se destina a este sector y realizando la comparativa entre los países latinoamericanos y México, para ello, se analiza la información estadística de carácter público obtenida de plataformas de instituciones tales como, el Instituto Nacional de Educación Educativa (INEE), el Centro de Investigación Económica y Presupuestaria (CIEP), la Organización para la Cooperación y el Desarrollo Económicos (OCDE), la Secretaría de Educación Pública (SEP). Los resultados obtenidos indican un aumento monetario significativo en el gasto ejercido en los últimos años en materia de educación.

Palabras clave: Gasto público, Producto Interno Bruto, Educación

Códigos JEL: I21, I22, H52.

\section{INTRODUCCIÓN}

La educación en la actualidad es un derecho humano que ayuda a desarrollar habilidades, aptitudes y actitudes para poder desenvolvernos en el mundo.

Este derecho está contemplado en la Declaración Universal de los Derechos

\begin{abstract}
The resources allocated to the education sector are an investment for Mexico because thanks to this the development of the country is promoted. In this article, the evolution of public spending in the education sector in Mexico is measured, taking as a point of comparison the percentage of Gross Domestic Product that is allocated to this sector and making the comparison between Latin American countries and Mexico, for this, it is analyzed public statistical information obtained from platforms of institutions such as the National Institute of Educational Education (INEE), the Center for Economic and Budgetary Research (CIEP), the Organization for Economic Cooperation and Development (OECD), the Secretariat of Public Education (SEP). The results obtained indicate an increase in significant monetary expenditure on education in recent years.
\end{abstract}

Keywords: Public spending, Gross Domestic Product, Education

JEL: I21, I22, H52.

Humanos (DUDH) proclamada por la Asamblea General de las Naciones Unidas en París, el 10 de diciembre de 1948 en su artículo $26^{\circ}$. (Asamblea general de las Naciones Unidas, 1948)

Antes de que fuera considerado un derecho humano fundamental, existía una gran 
segmentación de las personas que podían tener acceso a este servicio, abordando principalmente dos aspectos; las clases sociales el cual va de la mano con el aspecto económico y desigualdad de derechos y oportunidades entre hombre y mujeres. Esta situación se fue transformando en el acceso gratuito a la educación y la igualdad y equidad de género.

El rol que ahora juega la educación de calidad surge a raíz de los Objetivos Del Milenio (ODM) de la Organización Mundial de las Naciones Unidas (ONU) en donde se determina en su segundo apartado titulado: Lograr la enseñanza primaria universal, el cual se limitaba solamente en brindar el acceso a la educación primaria a todos los niños y niñas, el cual se enfocaba en la cobertura que se esperaba lograr para 2015 (ONU, 2000). Posterior a concluir la etapa de cumplimiento de los ODM se transformaron en los Objetivos de Desarrollo Sostenible (ODS) en donde se despliega en su objetivo cuatro: la Educación de Calidad en la cual se pretende garantizar una educación inclusiva y equitativa de calidad, así como promover oportunidades de aprendizaje permanente para la sociedad (ONU, 2015).

Girando en torno a estos dos ejes principales en donde se encuentra una estrecha relación entre el presupuesto destinado a la educación con la cobertura que las instituciones tanto públicas o privadas brindan a la población; por otro lado, la calidad será determinada por las instituciones facultadas bajo sus preceptos e indicadores que ponderan la calidad de la educación.

La educación es importante para el desarrollo social y económico de un país, pero para llegar a formar buenos profesionistas es necesario tener unas bases firmes, es decir, hacer buen uso de los recursos públicos destinados a la educación en el nivel básico principalmente.

En México a lo largo de 29 años la educación ha sufrido 5 reformas educativas, determinando que aproximadamente cada 6 años existe una nueva reforma, esto quiere decir que cada inicio de un nuevo sexenio el presidente electo decide proclamar una nueva. Esto conlleva que la reforma anterior no termine de madurar y exista un efecto negativo en la calidad de la educación además de tener un sesgo en la información recabada.

La presente investigación tiene como objeto medir la evolución del Gasto Nacional Educativo (GNE) de México a través del porcentaje destinado del Producto Interno Bruto al gasto educativo, además de hacer la comparativa con países Latinoamericanos y desglosar un análisis respecto al porcentaje del PIB que cada uno de estos aporta. Lo anterior para determinar las brechas que existe entre México y los países Latinoamericanos en materia de gasto en Educación.

Ampliar el acceso a la educación y las tasas de matriculación en las escuelas tiene relación directa con la educación de calidad la cual conlleva la ponderación de ciertos rubros los cuales pueden llegar a ser diferentes en los organismos internacionales, países, instituciones, etc.

Además, la educación de calidad es un concepto transversal con los otros 16 ODS, teniendo como finalidad el bien común cuyo eslogan de la ONU es que nadie se quede atrás (ONU, 2015).

Actualmente la educación está enfrentando la pandemia derivada por el SAR-COV-19 no solamente en México, sino también en el resto del mundo. La situación que estamos viviendo actualmente hace que las autoridades encargadas de la educación se pongan a trabajar para que el derecho a la educación se otorgue a todos los niños del país ya que actualmente debido a la pandemia y el no poder estar en contacto con las demás personas ha hecho que muchos niños, jóvenes no tengan acceso a la educación como es debido, esto gracias a que no todos tienen el acceso a una computadora, a internet.

Al hablar de educación pensamos en todo lo que conlleva; la infraestructura, los docentes, alumnos, directivos, aulas, reformas educativas, programas, planes de clase, material didáctico, la enseñanza: metodológica, social, cultural religioso, económico, psicológico, etc., es todo un mundo en donde el docente tiene que estar preparado y capacitado para poder ser un facilitador en el salón de clase, así poder apoyar a los estudiantes. 
La educación es parte fundamental para el desarrollo de un país y un derecho para todos los niños, niñas, jóvenes, adultos que quieran superarse. La educación, aparte de influir en el desarrollo de un país, también, enriquece la cultura, los valores y aporta grandes conocimientos a los estudiantes.

La educación en México, como ya se ha mencionado, es un derecho ya establecido en la constitución, por ello es indispensable que en la educación haya cambios, actualizaciones para que les brinden un servicio de calidad a todos aquellos que quieran aprender, que quieran superarse, para que adquieran nuevas habilidades y conocimientos que les ayuden en su vida diaria y a ejercer sus otros derechos. Esto será siempre a beneficio de la nación.

Hablar de la educación nos lleva a un mundo lleno de mucha información, donde cada nivel es un mundo totalmente diferente.

\section{MARCO TEÓRICO}

\subsection{Aspectos generales}

En este apartado comentamos algunos de los conceptos específicos.

El gasto público se entiende como aquella erogación que realiza el Estado para efectuar sus fines y sostener su estructura, los recursos que emplea provienen de las contribuciones que dan los ciudadanos de los impuestos (Chapoy, 1993). En donde el concepto de educación de acuerdo con la Secretaría de Educación Pública (SEP) la define como el principal componente del tejido social y el mejor instrumento para garantizar equidad y acceso a una mejor calidad de vida para todos, además de ser formadora del talento humano requerido para la competitividad y el desarrollo del país (SEP, 2020).

Según el artículo quinto de la Ley General de Educación se define la educación como:

"Es un medio para adquirir, actualizar, completar y ampliar sus conocimientos, capacidades, habilidades y aptitudes que le permitan alcanzar su desarrollo personal y profesional; como consecuencia de ello, contribuir a su bienestar, a la transformación y el mejoramiento de la sociedad de la que forma parte" (Cámara de Diputados, 2019, p.2).
En este sentido, el Estado garantizará la calidad en la educación obligatoria de manera que los materiales y métodos educativos, la organización escolar, la infraestructura educativa y la idoneidad de los docentes y los directivos garanticen el máximo logro de aprendizaje de los educandos (Cámara de Diputados, 2021a, p.5).

\subsection{Sistema Educativo Mexicano}

La Constitución Política de los Estados Unidos Mexicanos en su artículo tercero establece, desde 1917, que la educación básica es obligatoria para toda la población del país.

Todo individuo tiene derecho a recibir educación. El Estado Federación, Estados, Ciudad de México y Municipios-, impartirá educación preescolar, primaria, secundaria y media superior. La educación inicial, primaria y secundaria conforman la educación básica; ésta y la media superior serán obligatorias, la educación superior lo será en términos de la fracción $\mathrm{X}$ del presente artículo... (Cámara de Diputados, 2021a, p. 5).

El Sistema Educativo Nacional (SEN) está basado en el marco jurídico de la Constitución Política de los Estados Unidos Mexicanos (CPEUM) en sus artículos $3^{\circ}$ y $4^{\circ}$, y también en la Ley General de Educación (LGE) (Cámara de Diputados, 2019; Cámara de Diputados, 2021a).

La LGE establece que el SEN se compone por autoridades educativas, profesores, alumnos, programas, planes, métodos de enseñanza, materiales educativos, infraestructura educativa, las instituciones educativas de cada estado creadas por la descentralización e instituciones privadas autorizadas para impartir estudios (Cámara de Diputados, 2019).

En la figura 1 se muestra cómo se encuentra categorizado el SEN, el cual se divide en tres tipos de educación: básica, media superior y superior, los primeros dos tipos, la educación básica y la media superior, son obligatorias según la Constitución Política de los Estados Unidos Mexicanos 
(INEE, 2008).

Tabla 1. Esquema del Sistema Educativo Nacional

\begin{tabular}{|c|c|c|}
\hline & & ucativo Mexicano \\
\hline Tipo educativo & Nivel Educativo & Tipo de Servicio \\
\hline \multirow{11}{*}{ Educación Básica } & \multirow{3}{*}{ Preescolar } & General \\
\hline & & Indígena \\
\hline & & Comunitaria \\
\hline & \multirow{3}{*}{ Primaria } & General \\
\hline & & Indígena \\
\hline & & Comunitaria \\
\hline & \multirow{5}{*}{ Secundaria } & General \\
\hline & & Técnica \\
\hline & & Telesecundaria \\
\hline & & Comunitaria \\
\hline & & Para Trabajadores \\
\hline \multirow{3}{*}{ Educación Media Superior } & \multicolumn{2}{|c|}{ Profesional Técnico } \\
\hline & \multirow{2}{*}{ Bachillerato } & Bachillerato general \\
\hline & & Bachillerato tecnológico \\
\hline \multirow{5}{*}{ Educación Superior } & \multirow{2}{*}{ Licenciatura } & Educación normal \\
\hline & & Universitaria y tecnológica \\
\hline & \multirow{3}{*}{ Posgrado } & Especialidad \\
\hline & & Maestría \\
\hline & & Doctorado \\
\hline
\end{tabular}

Fuente: Elaboración propia basado en INEE (2008

El hacer obligatorio la educación media superior surge a partir del 9 de febrero del 2012 y propone alcanzar una cobertura total en todas las modalidades a más tardar en el ciclo escolar 2021 a 2022 (Presidencia de la
República de México, 2019).

El Sistema Educativo Nacional también se divide tres modalidades, escolarizada, no escolarizada y mixta, estas modalidades van acorde a los métodos de 
enseñanza, por ejemplo, la modalidad escolarizada es la que se imparte de manera presencial, ésta tiene una mayor cobertura en donde a cada alumno se le asigna un plantel y se cubre un plan de estudios el cual está programado conforme al calendario oficial de actividades, a comparación de la no escolarizada y la mixta, en estas modalidades se imparten como enseñanza abierta, es decir, a distancia de forma no presencial $o$ combinando las clases presenciales con la enseñanza a distancia (INEE, 2008).

Estas modalidades se adaptan a las necesidades de los estudiantes, es decir, las modalidades no escolarizada y mixta las toman quienes quieren estudiar, pero también necesitan trabajar o realizar distintas actividades las cuales no les dejan asistir a una escuela presencialmente. De igual manera estas modalidades se crearon para atender la demanda en las localidades donde no existían planteles escolares, implementaron el uso de tele sesiones a través de sesiones pregrabadas por profesores especializados en cada una de las materias que se imparten en los distintos niveles de educación. La modalidad no escolarizada está más enfocada en un sistema pedagógico basado en el "mostrar" que en el “enseñar" (INEE, 2008).

La SEP es el organismo que se encarga de la regulación y de la impartición de la educación en sus tres niveles (figura 1), también es responsable de las artes, la cultura y la investigación tanto científica como tecnológica (SEP, 2020).

A partir de la descentralización de los servicios educativos a las entidades federativas en 1993, existen organismos responsables de la educación en cada entidad federativa, estos organismos están encargados de brindar la educación inicial básica, especial y normal, también brindar capacitación, mantener actualizados a los profesores que conforman la educación básica (Instituto de Investigaciones Legislativas del Senado de la República, 2002).

Los cambios que se han implementado en el sistema educativo mexicano en los últimos 25 años han sido muy pocos, los cambios recientes ha sido la descentralización antes mencionada y la obligatoriedad de la secundaria en 1993, hacer obligatorio, también en preescolar de tres años en 2003 y por último hacer la educación media superior obligatoria en 2012 (Instituto de Investigaciones Legislativas del Senado de la República, 2002).

El 20 de abril del 2021, en el Diario Oficial de la Federación (DOF), se publicó la Ley General de Educación Superior (LGES), la cual fue aprobada por la Cámara de Diputados el 9 de marzo de 2021 (SEP, 2021).

La LGES ordena la creación de un sistema nacional de evaluación y un sistema nacional de información de la educación superior que permitirá el funcionamiento efectivo del Sistema Nacional de Educación Superior, que se conforma por tres subsistemas: universitario, tecnológico, educación normal y formación docente (Cámara de Diputados, 2021b).

\subsection{Calidad en educación}

La calidad en la educación está conformada por diversos factores, algunos de ellos no están dentro del sistema educativo, pero para poder brindar una educación de calidad se debe de proporcionar los conocimientos necesarios para que los estudiantes adquieran competencias, los aprendizajes, las habilidades, aptitudes y actitudes (INEE, 2018a).

La calidad del sistema educativo es la cualidad que resulta de la integración de las dimensiones de pertinencia, relevancia, eficacia interna, eficacia externa, impacto, suficiencia, eficiencia y equidad (INEE, 2018a)

Según los resultados de las pruebas nacionales realizadas en el 2017 dentro del Plan Nacional para la Evaluación de los Aprendizajes de la Secretaría de Educación Pública, 7 de cada 10 estudiantes tiene dificultades en la comunicación y en el lenguaje. Las escuelas comunitarias son las que presentan un grado más alto de marginación y son las que tuvieron el dominio más bajo de los programas educativos. De igual manera son las escuelas que tienen un grado más alto de deserción escolar ya que los estudiantes en ocasiones no tienen los 
recursos necesarios para seguir estudiando (INEE, 2018a).

De acuerdo con la UNICEF es importante fortalecer las capacidades de los profesores, tanto los que están en formación como los que están impartiendo clase, con capacitaciones que apoyen a su actualización, poniendo como prioridad las escuelas indígenas y las que se encuentran en zonas vulnerables (UNICEF, 2018).

México es el país de la OCDE que destina más recursos a la educación del gasto público total (18.4\%), pero en relación con el PIB está por encima del promedio de la OCDE (5.4\%) y términos de gasto por alumno es uno de los países que menos recursos destina a cada alumno (CIEP, 2016).

Existen diversos métodos de valuación del rendimiento académico de los alumnos en matemáticas, ciencia y lectura tanto nacionales como internacionales, a nivel internacional tenemos el Programa para la Evaluación Internacional de Alumnos (PISA, por sus siglas en inglés), en donde México está muy por debajo de los resultados deseados para una educación de calidad. Según la estandarización internacional PISA, en 2018 México se encuentra en el lugar 61 de 71 países evaluados, en una comparativa de países latinoamericanos. De acuerdo con datos de la OCDE, México se encuentra en el tercer lugar por debajo de Uruguay y Chile, pero aún da preocupación el lugar en el que se encuentra ubicado ya que nos muestra focos rojos para que las autoridades educativas diseñen nuevas estrategias para que mejore la enseñanza impartida a los estudiantes (OCDE, 2018).

La educación en México tiene varias deficiencias: es insuficiente, desigual y carece de calidad. Estas deficiencias se marcan más conforme el nivel de educación va incrementando, es decir, en nivel básica preescolar, primaria y secundaria estas deficiencias son nulas porque es obligatoria y el gobierno está obligado a proporcionar educación a todos los niños, jóvenes y adolescentes. Sin embargo, en la educación superior, es muy complicado que los estudiantes tengan la oportunidad de poder llegar a ser profesionistas (CONEVAL,
2018).

\subsection{Evolución del gasto público en México}

Invertir en la educación es parte importante para cualquier país porque están generando nuevos profesionistas que serán los que en un futuro moverán la economía del país. En el caso de América Latina y conforme a la UNESCO la inversión que realicen estos países tendrá que ser de al menos $6 \%$ de su PIB o $20 \%$ de su presupuesto público total (CIEP, 2021).

En México la inversión en educación ha rondado entre el $6.5 \%$ y el $6.9 \%$ del PIB, sin embargo, el GNE está por debajo del porcentaje que está establecido en la Ley General de Educación que es del 8\% (CIEP, 2021).

La evolución que se presenta en el sector educativo en México de \$492,094.70 millones de pesos en 2010 a $\$ 685,575.90$ millones en 2016, esto representa un incremento significativo, pero en comparación al porcentaje destinado al PIB en ejercicios anteriores el incremento es ligeramente menor. Para el año 2016 los recursos destinados a educación fueron el tercero más importante, por debajo del ámbito de energía y el gasto social (Cámara de Diputados, 2015).

México destina 2,598 dólares por alumno en comparación con Estados Unidos quien destina 14,269 dólares por alumno (Cámara de Diputados, 2015).

En la tabla 2 se muestra que los cambios del presupuesto de la SEP del 20202021 han sido drásticos en algunos rubros. Las disminuciones a los programas presupuestarios más significativos son: programa de igualdad entre mujeres y hombres SDN; programa para el desarrollo profesional docente; proyectos de infraestructura social del sector educativo; registro nacional de profesionistas y sus asociaciones; educación física de excelencia (CIEP, 2021).

De acuerdo con la tabla 2 los programas del nivel de educación superior, los que tuvieron incrementos son los siguientes: apoyos a centros y organizaciones de 
educación; diseño de política educativa, jóvenes escribiendo el futuro, la escuela es nuestra; normalización y certificación en competencias laborales (CIEP, 2021).

Tabla 2. Programas presupuestarios con mayores cambios 2020-2021

\begin{tabular}{lr}
\hline \multicolumn{1}{c}{ Programa } & Porcentajes \\
\hline Programa de igualdad entre mujeres y hombre SDN & -97.1 \\
\hline Programa para el desarrollo profesional docente & -48.8 \\
\hline
\end{tabular}

Proyectos de infraestructura social del sector educativo

Fuente: Elaboración propia basado en CIEP (2021).

\subsection{Presupuesto y gasto educativo}

El GNE tiene dos financiamientos: público y privado. El financiamiento público se refiere a los recursos destinados a educación, cultura, deporte, ciencia y tecnología; mientras que el financiamiento privado engloba los recursos de las inscripciones, colegiaturas, cuotas uniformes, materiales, transporte escolar que los usuarios de las instituciones tanto públicas como privadas desembolsan (INEE, 2018).

En el Proyecto de Presupuesto de Egresos de la Federación 2021 (PPEF 2021) el gasto educativo asciende a 836 mil 400 millones de pesos (mdp). Si se aprueba el gasto educativo a comparación del Presupuesto de Egresos de la Federación (PEF 2020) que fue de 807 mil 305 mdp, se tendría un crecimiento nulo en términos reales (CIEP, 2021).

El Proyecto de Presupuesto de Egresos de la Federación, asigna recursos a la educación en tres Ramos: el Ramo 11 es el que administra la SEP para todo el país; el Ramo 25 es utilizado para sostener el sistema educativo de la Ciudad de México; por último, algunos rubros del Ramo 33 son las participaciones que recibirán los demás 
estados para poder hacer frente en mayor o menor medida la educación en sus territorios (CIEP, 2021).

El $98.1 \%$ del presupuesto destinado a educación se usará para cubrir el gasto corriente, como es la nómina, gastos operativos, es decir, gastos para prestar servicios educativos. Mientras que el $1.8 \%$ será destinado para cubrir gastos de obra pública tales como compra, alquiler o mantenimiento de bienes muebles. El $0.02 \%$ a gasto corriente por concepto de gastos indirectos de programas subsidiarios, y el $0.03 \%$ a la construcción de obra pública (CIEP, 2021).

A la educación básica se destina el $61.2 \%$ del presupuesto, mientras que a la educación superior donde se ubica el $9.4 \%$ de la matrícula se destinará $16.6 \%$ de los recursos; a la educación media superior se asignará el $14.4 \%$ del gasto del año. Al posgrado se destinará el $1 \%$ del gasto educativo 2021. El $6.8 \%$ de los recursos restantes se enfocarán a las otras subfunciones del gasto educativo (CIEP, 2021).

\section{MÉTODO}

La metodología utilizada en esta investigación por sus características corresponde a un enfoque del tipo cualitativa con un alcance exploratorio. Además, es documental porque los datos se obtuvieron a través de la revisión de diversas páginas de instituciones como el Instituto Nacional de Educación Educativa (INEE), el Centro de Investigación Económica y Presupuestaria (CIEP), la Organización para la Cooperación y el Desarrollo Económicos (OCDE) la Secretaría de Educación Pública (SEP), entre otras.

Una vez recabada la información, se procedió al análisis de datos con el propósito de observar el comportamiento de las variables estudiadas $\mathrm{y}$ obtener mayor objetividad en relación con el objetivo y las premisas planteadas en esta investigación.

\section{RESULTADOS}

A continuación, se muestra un análisis de comparación de diferentes indicadores importantes para el rubro de educación.

Figura 1. Crecimiento del gasto público por alumno y matrícula

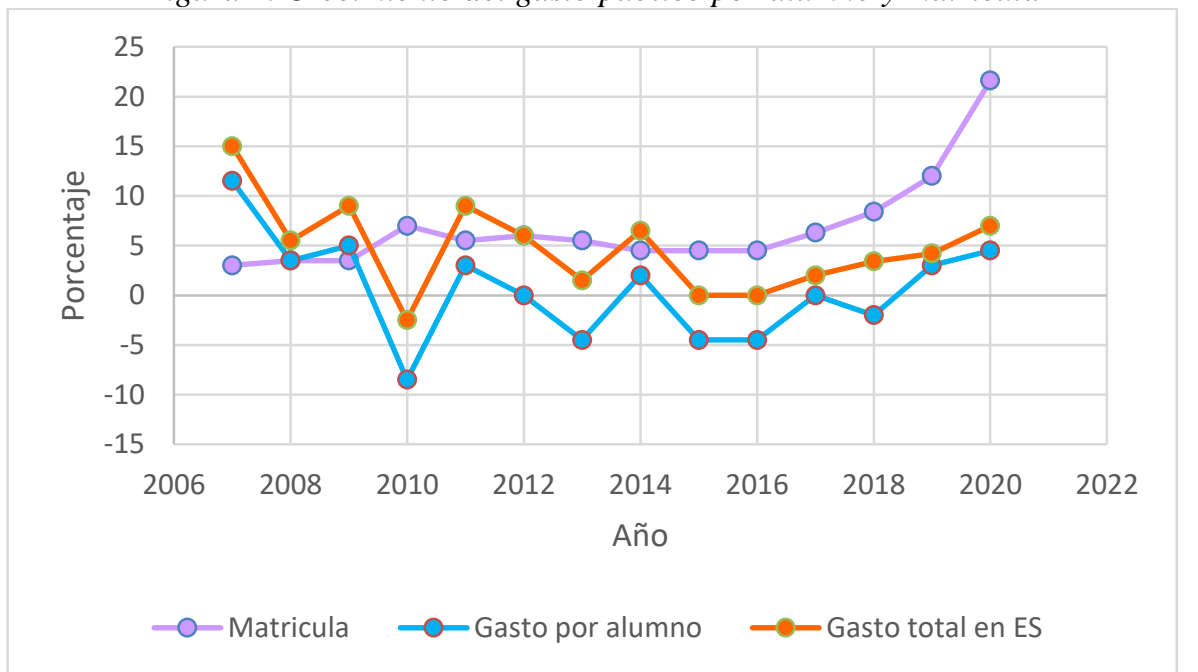

Fuente: Elaboración propia con datos de las Cuentas Públicas 2006-2020 (SHCP, 2006-2020); del PEF 2020 (Cámara de Diputados, 2020); y el Sistema de Indicadores Educativos (Dirección General de Planeación, Programación y Estadística Educativa, SEP, (2020).

En la figura 1 se muestra la variación de los porcentajes por alumno y matrícula en los últimos 15 años para tener un panorama más amplio respecto al movimiento que ha tenido. Asimismo, se observa que ha estado constante con algunos piques, se tuvo un decremento en el año 2010 esto debido a la crisis del 2009 que, de acuerdo con expertos, ha sido la peor recesión económica que ha tenido el país en 70 años. Anterior a esto, en 2008 el peso tuvo 
varias devaluaciones lo cual provocó que perdiera alrededor del $50 \%$ de su valor frente al dólar, esto provocó que el secretario de educación de este tiempo, Alonso Lujambio, solicitará a las universidades públicas del país que pidieran una reducción de sus gastos de manera "voluntaria", es por ello que en la figura 1 se muestra la disminución en 2010 .
Otro pico importante que vemos en la figura 1 es el del 2014 en donde vemos un incremento, uno de los cambios que hubo en ese año y que influyó en el incremento del gasto fue la creación del Programa Nacional de Becas, en el que se agrupan varios programas que anteriormente eran independientes.

Figura 2. Gasto público mundial en educación, total (\% del PIB)

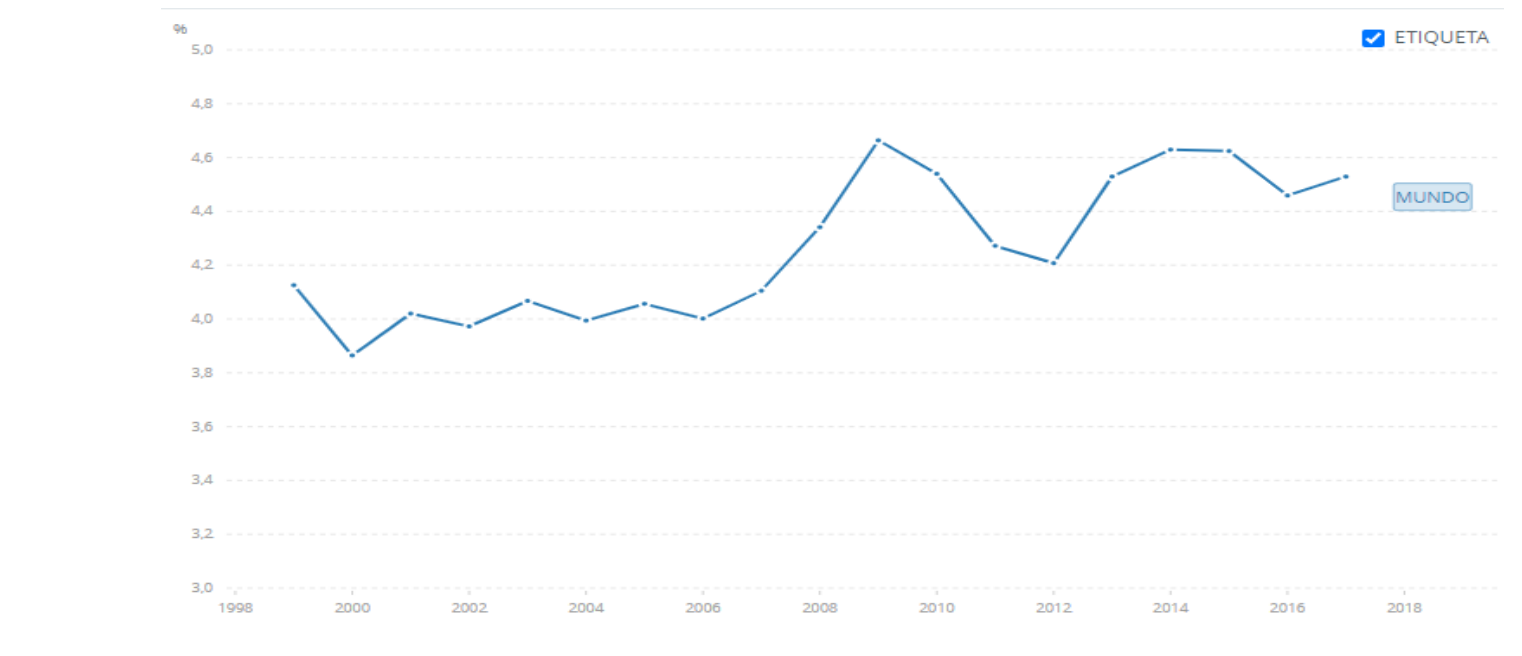

Fuente: Banco Mundial (2017)

De acuerdo con datos del Banco Mundial, se ha tenido un incremento gradual desde 2000 hasta 2009 donde precisamente en este año se obtuvo un $4.66 \%$ del PIB mundial destinado al gasto público, siendo este el mayor porcentaje registrado hasta ahora. A partir de este año se presentó un decremento gradual hasta 2012 de $4.20 \%$ y posteriormente se obtuvo de nuevo un incremento progresivo (ver detalle en la figura 2).
Respecto al porcentaje del PIB que América Latina destina para el presupuesto a la educación, podemos observar un crecimiento bastante bajo pero que ha ido de manera paulatina desde 2000 hasta 2009 teniendo un comportamiento bastante similar al PIB mundial, cuyo promedio fue de $3.77 \%$ en el transcurso de 2000 a 2018. 


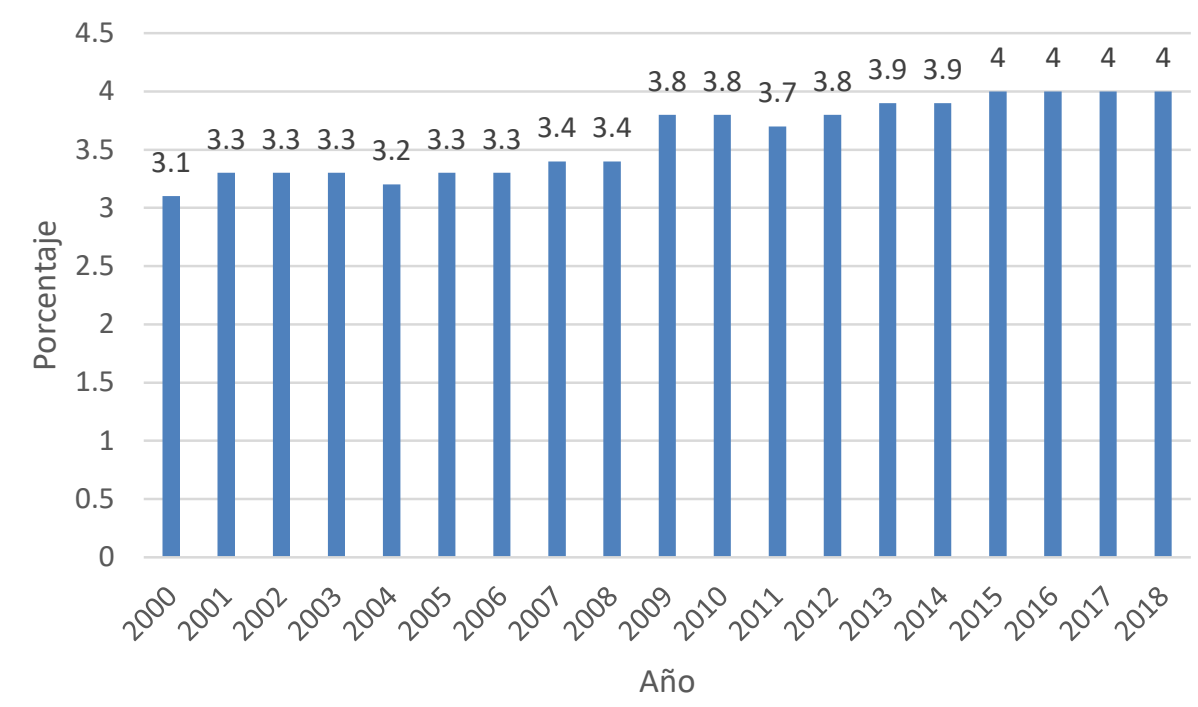

Fuente: Elaboración propia con datos de CEPAL (2018).

Respecto al promedio del PIB destinado a la educación por parte de América Latina, en los últimos 10 años ha sido del $3.9 \%$. El porcentaje más alto obtenido en los últimos 10 años fue en 2011 con un $3.7 \%$ estando debajo solo por $0.3 \%$ puntos con respecto al porcentaje más alto obtenido que fue de 2015 a 2018 manteniéndose con un $4 \%$ (ver detalle en la figura 3).

Figura 4. Gasto público en América Latina en educación (\% del PIB)

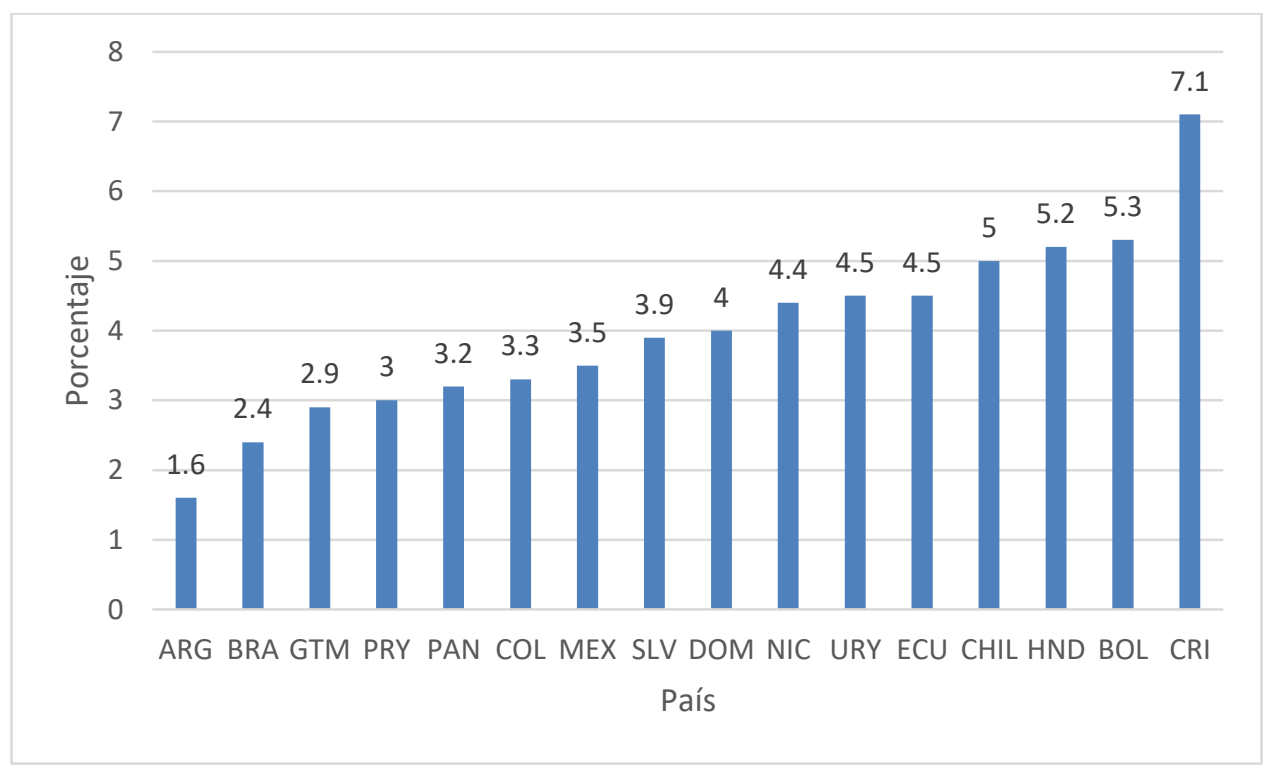

Fuente: Elaboración propia con datos de CEPAL (2019).

Respecto a la comparación de algunos países de América Latina podemos observar que México se encuentra en el $7^{\circ}$. lugar con un $3.5 \%$ de los países que destinan menos porcentaje de su PIB a la educación, siendo Argentina 
el país que menos destina con un $1.6 \%$ y Costa Rica el más alto con un $7.1 \%$ (ver figura 4).

Figura 5. Gasto Público en Educación, total (\% del PIB)

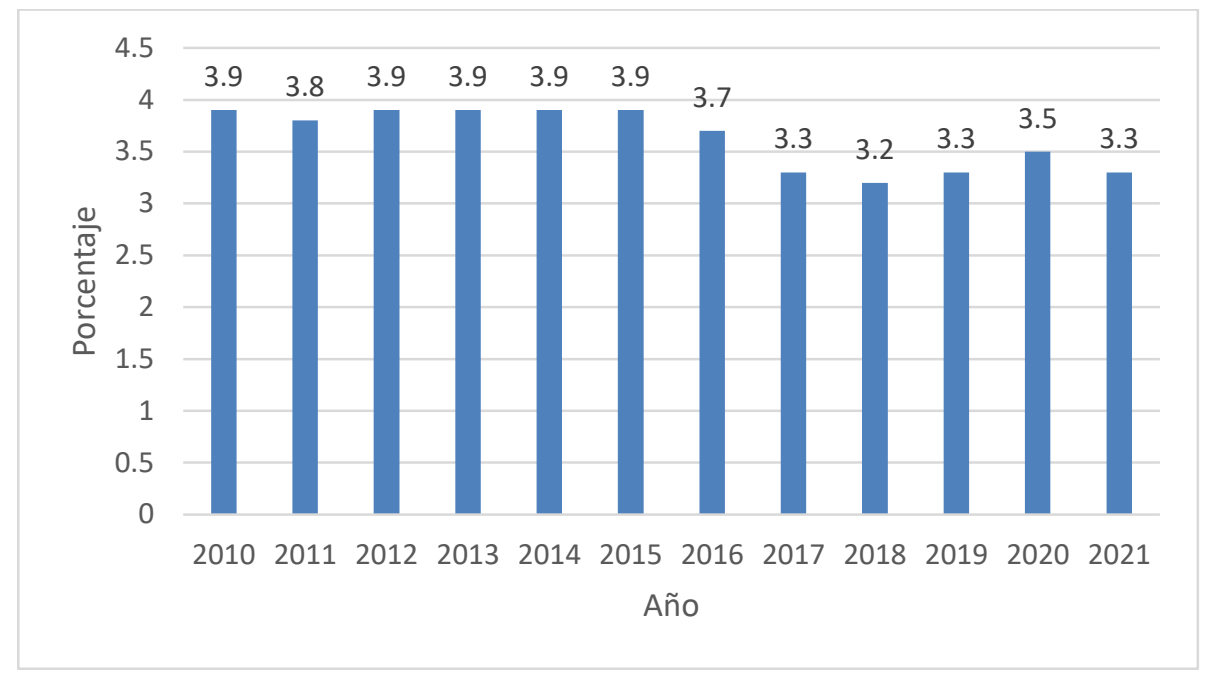

Fuente: Elaboración propia con datos de SHCP (2021).

De acuerdo con la figura 5 en los últimos 10 años México ha tenido un promedio de $3.63 \%$ de su PIB destinado al rubro de educación. En 2018 tuvo el porcentaje más bajo con un $3.2 \%$, mientras que el porcentaje más alto de 2010 a 2015 fue de $3.9 \%$ en dicho periodo.

El comportamiento del PIB en México con respecto al GNE ha sido un tema relevante, ya que en los últimos 12 años se ha visto un decremento paulatino en el porcentaje destinado para este rubro, teniendo su declive más significativo en 2017 al disminuir de 3.7 a 3.3, manteniendo una estabilidad hasta 2019 y un incremento de nuevo en 2020 de $3.5 \%$ (ver figura 5).

\section{CONCLUSIONES}

De acuerdo con la información recabada se puede concluir que el GNE en México ha tenido un crecimiento positivo, ya que en el transcurso del tiempo ha tenido un incremento monetario constante en el gasto ejercido, pero también presenta variaciones constantes y una tendencia a la baja, esto quiere decir, que si bien el gasto al final del periodo es mayor que al inicio, el presupuesto que se destina a la educación ha disminuido. Dadas las evaluaciones realizadas tanto a nivel nacional como internacional, México es un país con muchas necesidades y esto requiere mucho trabajo para poder identificar los instrumentos necesarios para mejorar la calidad y hacer un mejor uso de los recursos.

El sistema educativo mexicano tiene grandes deficiencias y gran parte para la mejora en cuanto a términos de calidad es necesario un incremento de la inversión para la capacitación y remuneración justa de los profesores, la infraestructura escolar, el tener cobertura para todos los que se encuentran en edad escolar en el país.

Al analizar el comportamiento del GNE se observa que representa el $13.69 \%$ del total del Presupuesto de Egresos de la Federación para 2020 (PEF), este rubro es un eje central del Plan de Desarrollo del país.

En la actualidad por los recortes presupuestales que ha realizado el gobierno actual, han reducido el presupuesto destinado a programas importantes para mejorar la educación en México teniendo una fuerte 
repercusión negativa, tales como, el presupuesto para la inclusión y la equidad educativa, el recorte fue del $38.6 \%$ siendo este concepto cada vez más tomado en cuenta en los planes educativos del Gobierno Federal, sin embargo, un recorte del $12.7 \%$ al programa de escuelas de tiempo completo, el programa de inglés sufrió un recorte de $53.4 \%$, y por último el programa de fortalecimiento a la calidad educativa que sufrió un recorte del $46.2 \%$.

Finalmente, analizando la información recabada en la presente investigación y para conseguir resultados positivos en la mejora de la educación en México y brindar una buena calidad necesitamos de mejores políticas educativas, en donde se necesitarán los recursos para poder operar con una planeación más efectiva para poder ofrecer una mejor educación a los estudiantes y que obtengan mejores aprendizajes. Mejorar el uso de los recursos públicos que se destinan al sector educativo se hará realidad cuando las autoridades ataquen el problema de raíz y hagan uso de la información disponible para diseñar programas que mejoren la calidad del sector educativo. 


\section{REFERENCIAS}

Banco Mundial BIRF - AIF (2017), Gasto público mundial en educación, total (\% del PIB). Disponible https://datos.bancomundial.org/indicator/SE.XPD.TOTL.GD.ZS?end=2019\&start=1998\&vie $\underline{w}=$ chart

Cámara de Diputados del H. Congreso de la Unión. (2015). Evaluación del gasto educativo en México. http://www5.diputados.gob.mx/index.php/esl/content/download/31549/158531/file/Reporte9 Evaluacion\%20Gasto\%20Educativo.pdf

Cámara de Diputados del H. Congreso de la Unión. (2019). Ley General de Educación. Disponible en http://www.diputados.gob.mx/LeyesBiblio/pdf/LGE_300919.pdf

Cámara de Diputados del H. Congreso de la Unión. (2021a). Constitución Política de los Estados Unidos Mexicanos. Disponible en http://www.diputados.gob.mx/LeyesBiblio/pdf_mov/Constitucion_Politica.pdf

Cámara de Diputados del H. Congreso de la Unión. (2021b). Ley General de Educación Superior. Disponible https://www.dof.gob.mx/nota_detalle.php?codigo $=5616253 \&$ fecha=20/04/2021

Catarsis. (2020). En Real Academia Española (Ed.) Diccionario de la lengua española (23a ed.). Disponible en https://dpej.rae.es/lema/producto-interior-bruto

Chapoy, B. (1993). Diccionario Jurídico Mexicano, Instituto de Investigaciones Jurídicas UNAM, Porrúa, México. Tomo II.

Centro de Investigación Económica y Presupuestaria, CIEP. (2016). El sistema educativo mexicano, rezagado en calidad y distribución de recursos. Disponible en: https://ciep.mx/el-sistemaeducativo-mexicano-rezagado-en-calidad-y-distribucion-de-recursos/

Centro de Investigación Económica y Presupuestaria, CIEP. (2021). Gasto público en educación. PPEF 2021. Disponible en: https://ciep.mx/gasto-publico-en-educacion-ppef-2021/

Comisión Económica para América Latina y el Caribe, CEPAL. (2018). Gasto en Educación. Disponible en https://observatoriosocial.cepal.org/inversion/es/indicador/gasto-educacion

Comisión Nacional de Evaluación de la Política de Desarrollo Social, CONEVAL. (2018). Estudio Diagnóstico del Derecho a la Educación 2018. Disponible en https://www.coneval.org.mx/Evaluacion/IEPSM/Documents/Derechos_Sociales/Estudio_Dia g Edu 2018.pdf

Fondo de las Naciones Unidas para la Infancia, UNICEF. (2018). Calidad Educativa. Disponible en: https://www.unicef.org/mexico/calidad-educativa

Instituto Nacional para la Evaluación de la Educación, INEE. (2008). Panorama Educativo de México. Indicadores del Sistema Educativo Nacional. Disponible en https://historico.mejoredu.gob.mx/wp-content/uploads/2018/12/P1B106.pdf

Instituto Nacional para la Evaluación de la Educación, INEE. (2018a). ¿Qué es la calidad educativa? Directrices para mejorar. Disponible en: https://historico.mejoredu.gob.mx/directrices-paramejorar/que-es-la-calidad-educativa/

Instituto Nacional para la Evaluación de la Educación, INEE. (20018b). Panorama Educativo de México. Indicadores del Sistema Educativo Nacional. Disponible en https://www.inee.edu.mx/wp-content/uploads/2019/08/P1B117.pdf

Instituto de Investigaciones Legislativas del Senado de la República, IILSEN. (2002). Evolución del gasto en educación en México. Disponible en http://chamilo.cut.edu.mx:8080/chamilo/courses/POLITICASPUBLICAYEDUCACION/doc ument/UNIDAD 2.7/Evoluciondelgastoeneducacion.pdf

Organización para la Cooperación y el Desarrollo Económicos, OCDE. (2018). Programa para la evaluación internacional de alumnos (PISA) PISA 2018, resultados. Disponible en https://www.oecd.org/pisa/publications/PISA2018_CN_MEX_Spanish.pdf 
Robles, V.H. (2009). Panorama educativo de México. Estructura y Dimensión del Sistema Educativo Nacional. INEE. México. 35-53

Secretaria de Educación Pública, SEP. (2020). Sistema Interactivo de Consulta de Estadística Educativa. Disponible en https://www.planeacion.sep.gob.mx/principalescifras/

Secretaria de Educación Pública, SEP. (2020) Secretaría de Educación Pública, Misión y Visión de la SEP. Disponible en https://www.gob.mx/sep/acciones-y-programas/vision-y-mision-de-lasep

Secretaria de Educación Pública, SEP. (2021). Publicación de la Ley General de Educación Superior en el DOF. Disponible en: https://www.educacionsuperior.sep.gob.mx/comunicados/2021/200421.html

Secretaria de Hacienda y Crédito Público, SHCP. (2019). Cuenta Pública 2019. Disponible en https://www.cuentapublica.hacienda.gob.mx/es/CP/2019

Secretaria de Hacienda y Crédito Público, SHCP. (2020). Presupuesto de Egresos de la Federación 2020. Disponible en https://www.transparenciapresupuestaria.gob.mx/es/PTP/Datos_Abiertos

Secretaria de Hacienda y Crédito Público, SHCP. (2021). Proyecto de Presupuesto de Egresos de la Federación (PPFE) 2021. Disponible en https://www.finanzaspublicas.hacienda.gob.mx/es/Finanzas_Publicas/Paquete_Economico_y Presupuesto

Sistema de Información de Tendencias Educativas en América Latina, SITEAL. (2019). Ley General de Educación. Disponible en https://siteal.iiep.unesco.org/bdnp/350/ley-generaleducacion\#: :text=Regula\%201a\%20educaci\%C3\%B3n\%20que\%20imparten,en\%20el\%20si stema\%20educativo\%20nacional 